\title{
Consumo de tabaco entre universitários da área de saúde
}

\author{
Mariana Godói Ferreira*, Jéssyca Reis Faria*, Beatriz Barco Tavares, D.Sc.**, \\ Luciano Garcia Lourenção, D.Sc.***
}

*Acadêmicas do Curso de Graduação em Enfermagem da Faculdade de Medicina de São José do Rio Preto-FAMERP, **Professora do Departamento de Enfermagem Especializada da Faculdade de Medicina de São José do Rio Preto-FAMERP, ***Professor do Departamento de Epidemiologia e Saúde Coletiva da Faculdade de Medicina de São José do Rio Preto-FAMERP

Pesquisa vinculada ao Núcleo de Estudos sobre Morbidade Referida e Processo de Gestäo em Saúde nas Diferentes Fases e Contextos de Vida Humana (NEMOREGES) da Faculdade de Medicina de São José do Rio Preto-FAMERP

\begin{abstract}
Resumo
Objetivo: Caracterizar o consumo de tabaco e determinar fatores relacionados ao consumo entre os universitários da área da saúde de Instituiçóes de Ensino Superior (IES) do interior do estado de São Paulo. Métodos: Estudo quantitativo, descritivo, com acadêmicos dos Cursos de Graduaçáo em Enfermagem e Medicina de Instituiçóes de Ensino Superior Pública e Privada de São José do Rio Preto-SP. O instrumento aplicado para coleta de dados foi adaptado do Inquérito Domiciliar sobre Comportamentos de Risco e Morbidade Referida de Doenças e Agravos não Transmissíveis do Instituto Nacional do Câncer. Os dados foram analisados com o Statistical Package for Social Sciences, versão 17.0. Resultados: Dos 369 acadêmicos, $73,7 \%$ eram do sexo feminino; a média da idade foi de 22,3 $\pm 5,13$ anos; $93,5 \%$ eram solteiros; $53,7 \%$ moravam com a família e $74,8 \%$ náo exerciam atividade remunerada. A tentativa de fumar/experimentar cigarros ao menos uma ou duas tragadas foi declarada por 43,9\% universitários; 8,7 \% haviam iniciado o consumo de tabaco há 11 meses, no período de graduação; $10,0 \%$ dos acadêmicos eram tabagistas e, destes, $61,8 \%$ fumavam cigarros diariamente; $79,3 \%$ dos fumantes eram do sexo feminino, $68,0 \%$ fumavam após a $1^{\text {a }}$ hora ao acordar; $70,0 \%$ fumavam há mais de 4 anos; $46,3 \%$ conviviam com uma ou mais pessoas tabagistas, sendo $16,5 \%$ amigos; e $1,1 \%$ ex-fumantes. Conclusão: É importante intensificar os programas antitabagismo entre os universitários para melhorar sua qualidade de vida e conscientizá-los da sua missão futura que é promover a saúde da população.
\end{abstract}

Palavras-chave: hábito de fumar, uso de tabaco, estudantes. 


\section{Abstract \\ The tobacco use among university students in the health professions}

Aim: To characterize tobacco use and to determine factors related to such use among university students in the health of Higher Education Institutions (HEIs) in the state of São Paulo. Methods: Quantitative, descriptive and prospective study with 369 students of Nursing and Medicine in public and private HEIs in São José do Rio Preto, mean age of 22 years, SD 5.13, 73.7\% were female, $93.5 \%$ single $53.7 \%$ lived with family and $74.8 \%$ without paying activity. The instrument used was adapted from the Household Survey on Risk Behavior and Morbidity of non-communicable Diseases of the National Cancer Institute. To analyze the data collected we used the Statistical Package for Social Sciences, version 17.0. Results: Attempt to smoke / experiment with smoking at least one or two puffs was reported by $43.9 \%$ university students; $8.7 \%$ started smoking 11 months ago, during graduation course; $10.0 \%$ of the students were currently smokers, of whom $61.8 \%$ smoke daily cigarettes; $79.3 \%$ of smokers were female and $20.7 \%$ male; $68.0 \%$ smoked after a first hour upon waking; $70.0 \%$ smoked for more than four years, $46.3 \%$ described one or more smokers in their social life, $16.5 \%$ were friends and $1.1 \%$ were former smokers. Conclusion: It is important to step up anti-smoking programs among the university students to improve their quality of life and make them aware of their future mission that is to promote health of population.

Key-words: smoking, tobacco use, students.

\section{Resumen}

\section{El consumo de tabaco entre los estudiantes universitarios en el área de la salud}

Objetivo: Caracterizar el consumo de tabaco y determinar los factores relacionados con el consumo entre los estudiantes universitarios del área de la salud de Instituciones de Educación Superior (IES) en el estado de Sáo Paulo. Métodos: Estudio cuantitativo, descriptivo, con académicos de Enfermería y Medicina de la Educación Superior en instituciones públicas y privadas de São José de Rio Preto - SP. El instrumento utilizado para la recolección de datos fue adaptado de la Encuesta de Hogares sobre Comportamiento de Riesgo y Enfermedades no Transmisibles del Instituto Nacional del Cáncer. Los datos fueron analizados con el programa Statistical Package for Social Sciences, versión 17.0. Resultados: De los 369 estudiantes, el 73,7 \% eran mujeres; la edad media fue de 22,3 \pm 5,13 años; 93,5 \% eran solteros; 53,7 \% vivían con la familia y el 74,8 \% no ejercían actividad remunerada. El 43,9\% de los universitarios declararon que intentaron inhalar al menos una o dos bocanadas de humo; 8,7 \% había comenzado a fumar hace 11 meses, en el período de la graduación; el 10,0 \% de los estudiantes eran fumadores y de éstos, el 61,8 \% fumaba cigarrillos cada día; 79,3\% de los fumadores eran mujeres, el 68,0 \% fumaban una hora después de despertarse; $70.0 \%$ fumaba hacía más de cuatro años; $46.3 \%$ convivían con alguien o más personas que fumaban, el 16,5 \% amigos; y el 1,1 \% eran exfumadores. Conclusión: Es importante intensificar los programas de lucha contra el tabaquismo entre los estudiantes universitarios para mejorar su calidad de vida y que sean conscientes de su futura misión que es la de promover la salud de la población.

Palabras-clave: hábito de fumar, uso de tabaco, estudiantes.

\section{Introdução}

O uso do tabaco mata ou incapacita muitas pessoas em seus anos mais produtivos, consome os orçamentos familiares, eleva o custo dos cuidados de saúde e dificulta o desenvolvimento econômico [1]. O tabagismo causa dependência química, e é uma doença crônica progressiva, extremamente democrática, pois afeta pessoas de qualquer idade, não importando o nível socioeconômico ou intelectual [2]. O número de mortes causado por doenças associadas ao consumo de tabaco é superior à soma das mortes por Aids, cocaína, heroína, álcool, acidentes de trânsito, incêndios e suicídios [1-3].

Segundo a Organização Mundial da Saúde (OMS), existem 1,3 bilhôes de fumantes no mundo e cerca de 4,9 milhóes de pessoas morrem anualmente em decorrência do tabaco. Durante o século XXI, a epidemia do tabaco matará, potencialmente, cerca de um bilhão de pessoas, se as tendências atuais de consumo continuar do mesmo modo, ou seja, matando cerca da metade de todos seus usuários $[1,4]$. Dentre as doenças tabaco-relacionadas que mais contribuem para a mortalidade estáo o câncer 
de traqueia/brônquios/pulmão, a doença isquêmica do coração, a doença pulmonar obstrutiva crônica (DPOC) e as doenças cerebrovasculares [2,5]. Desde o fim da década de 1970, diversos autores passaram a relacionar também o tabagismo a aspectos negativos da afetividade, tais como tensão, ansiedade, irritabilidade, humor e sintomas depressivos em geral [6].

Estima-se que em 2020, para cada 10 mortes atribuídas ao tabaco, 7 ocorrerão em países em desenvolvimento, devido ao baixo nível de informação da população sobre os riscos do tabaco e à alta aceitação social, influenciada pelas estratégias de marketing das empresas de tabaco, direcionadas principalmente a população jovem [2,3]. Segundo a OMS, o tabagismo é uma doença pediátrica, já que quase $90 \%$ dos fumantes regulares começam a fumar antes dos 18 anos [4]. O marketing das indústrias do fumo para manter e expandir suas vendas tem como foco os jovens, pois o tabaco é a segunda droga mais consumida entre eles, no mundo e no Brasil, decorrente das facilidades e estímulos para obtenção do produto, com destaque para o baixo custo $[1,4]$.

Paralelamente a este quadro, constata-se que o ingresso na universidade, ainda que traga sentimentos positivos e de alcance de uma meta programada, por vezes pode se tornar um período crítico, de maior vulnerabilidade para o início e a manutenção do uso de drogas [7]. Os alunos iniciam o uso de alguma droga na companhia de colegas da faculdade, com quem geralmente estabelecem laços emocionais e compromissos importantes que facilitam e incentivam esse hábito em festas e ritos de iniciação. Esta influência é definida como uma norma, como "um atributo que descreve e prescreve um grupo social" [8]. Apesar dos acadêmicos descreverem o entretenimento como um fator de proteçáo para minimizar o estresse e se distanciarem dos problemas do dia-a-dia, esta atitude indica que as drogas, principalmente as lícitas, exercem um forte atrativo para o grupo, em função de sua maior tolerância, por serem aceitas socialmente e pelo fácil acesso [810]. Além disso, existe a hipótese de uma escalada de drogas lícitas para ilícitas, afetando severamente o estado de saúde [11].

De maneira geral, as condutas de saúde entre os estudantes do início e do final da graduação não apresentam diferenças significativas, mas observa-se o aumento dos hábitos nocivos, como tabagismo, no final do curso [12].

Diante desta problemática, cientes de que os universitários da área de saúde serão formadores de opiniáo junto à população, cuidadores e educadores de saúde, este trabalho objetivou caracterizar o consumo de tabaco e determinar fatores relacionados a esse consumo entre os universitários da área de saúde em Instituiçóes de Ensino Superior de São José do Rio Preto/SP.

\section{Material e métodos}

Estudo prospectivo e descritivo com abordagem quantitativa, aprovado pelo Comitê de Ética em Pesquisa da Faculdade de Medicina de São José do Rio Preto (FAMERP), parecer no. 067/2010, segundo as normas regulamentares n. 196/96, para pesquisas com seres humanos.

Participaram 369 (100,0\%) acadêmicos, destes 269 (72,9\%) dos cursos de Graduação em Enfermagem e Medicina de Instituiçóes de Ensino Superior (IES) pública e $100(27,1 \%)$ da Escola privada de Sáo José do Rio Preto, a noroeste do Estado de São Paulo.

Esclarecemos, ainda, que participaram apenas alunos da $1^{\mathrm{a}}$ à $4^{\mathrm{a}}$ série do curso de Graduação de Medicina, pois os acadêmicos da $5^{\mathrm{a}}$ e da $6^{\mathrm{a}}$ séries participam do internato, uma atividade extraclasse.

A coleta de dados foi realizada em novembro de 2010, em sala de aula, após consentimento do docente responsável. O pesquisador esclarecia aos acadêmicos o objetivo da pesquisa, entregava o Termo de Consentimento Livre Esclarecido (TCLE) (duas vias, uma para o participante e a outra para o pesquisador) e os que aceitavam participar, após a assinatura do TCLE, recebiam o questionário composto por questóes sobre as condiçóes socioeconômicas, consumo de álcool e qualidade de vida para ser respondido imediatamente. Ao término, o aluno depositava o questionário em uma caixa para garantir o sigilo e anonimato.

O instrumento de coleta de dados deste estudo foi adaptado do Inquérito Domiciliar sobre Comportamentos de Risco e Morbidade Referida de Doenças e Agravos não Transmissíveis do Instituto Nacional do Câncer (INCA) [13]. O instrumento de coleta teve como base os questionários do Projeto Carmen, da Organização Mundial da Saúde/ OPAS, WHO Standard Risk Factor Questionnaire [14], Behavioral Risk Factor Surveillance System (BRFSS-CDC) [15], Third Health and Nutrition Examination Survey (USDHHS) [16] e International Physical Activity Questionnaire Young and Middle-aged Adults (IPAQ) [17] e aborda as se- 
guintes variáveis: situação e exposição ocupacional, exposição solar, atividade física, dieta, tabagismo, álcool, percepção de saúde e morbidade referida, pressão arterial, colesterol, diabetes, câncer, qualidade de vida - condição funcional, exames para detecção de câncer de colo de útero e mama e uso de hormônios, violência doméstica, acidente de trânsito individual. Esse inquérito é disponibilizado pelo INCA para utilização em pesquisas científicas e, para este estudo, selecionaram-se as variáveis: álcool e qualidade de vida - condiçáo funcional.

As questóes sobre o consumo de tabaco direcionaram uma estimativa de tabagismo ativo e de exposição tabagística ambiental, para descrição do perfil do fumante, além de variáveis relativas à cessaçáo do hábito de fumar, opinióes, conhecimentos e atitudes quanto à exposição tabagística ambiental.

Os dados foram armazenados em planilha do Excel $^{\circledR} 2007$ e analisados pelo programa Statistical Package for Social Sciences (SPSS), versão 17.0. Para tanto, foram agrupados por especificidade, analisados e apresentados em tabelas ou de forma descritiva, em valores absolutos e percentuais, com análise da média \pm Desvio Padrão (DP), quando pertinente, considerando significante valor $\mathrm{p} \leq 0,05$.

\section{Resultados}

Participaram 369 acadêmicos, 269 (72,9\%) cursavam período integral em uma IES que é autarquia estadual e $100(27,1 \%)$ estudavam meio período em uma IES particular. Os acadêmicos tinham uma média de idade de $22,3 \pm 5,13$ anos; $73,7 \%$ eram do sexo feminino; $92,7 \%$ solteiros e $78,0 \%$ residiam em São José do Rio Preto-SP. Verificou-se que $53,7 \%$ moravam com a família, $35,0 \%$ com os amigos e $11,3 \%$ sozinhos; $98,4 \%$ declaravam ter uma religião; destes, $62,6 \%$ eram católicos. Estavam distribuídos da seguinte forma: $72,6 \%$ no curso de enfermagem e $27,4 \%$ no de medicina.

$\mathrm{O}$ investimento do curso na IES particular era autofinanciado por $60,2 \%$ dos acadêmicos, ou seja, pago integralmente pelo estudante ou família sem auxílio de bolsa de estudo; 31,6\% recebiam o auxílio da família para viver e $8,2 \%$ possuíam bolsa de estudo integral. Descobriu-se também que 74,8\% não exerciam atividade remunerada, pois na IES pública os cursos aconteciam em período integral. A renda econômica de 33,1\% dos universitários era de 2 a 3 salários mínimos.

\section{Consumo de tabaco}

A tentativa de fumar/experimentar cigarros, ao menos uma ou duas tragadas, foi declarada por 162 (43,9\%) universitários. Dentre esses, 22,8\% declararam já ter fumado ao menos 5 maços ou 100 cigarros durante sua vida. O tempo de consumo regular em anos, ou seja, pelo menos 1 cigarro por semana (considerando aqueles que já cessaram o hábito) foi de 1 a 5 anos para 50,0\% dos alunos; de 6 a 10 anos para 36\%; de 11 a 16 anos para $13,15 \%$ dos alunos. Destaca-se, ainda, que 8,7 \% iniciaram o consumo de tabaco há 11 meses, ou seja, no período de graduaçáo.

Verificou-se que 10,0\% dos acadêmicos fumavam. Desses, 61,8\% fumavam diariamente, sendo que $80,0 \%$ fumavam de 1 a 6 cigarros e $20 \%$ de 10 a 20 cigarros. $\mathrm{O}$ cigarro industrializado com filtro foi o tipo mais utilizado e $54,3 \%$ fumavam cigarros com baixos teores/suave/light.

Apesar da variedade de cigarros industrializados, 63,6\% dos usuários de tabaco utilizavam sempre uma mesma marca e $36,4 \%$ consumiam o cigarro independente da marca. Entre os tabagistas, $93,7 \%$ compravam o maço de cigarros e $6,3 \%$ compravam apenas a unidade.

Ao analisar o tempo do consumo do primeiro cigarro após acordar, constatou-se que 20,0\% usavam nos primeiros 5 minutos, $8,0 \%$ de 6 a 30 minutos, $4,0 \%$ após 30 minutos e $68,0 \%$ fumavam após a primeira hora.

Em relação ao consumo de tabaco por sexo, constatou-se que $79,3 \%$ dos fumantes são do sexo feminino e $20,7 \%$ masculino. Outras características relativas ao consumo de produtos derivados de tabaco como os cigarros indianos, de bali, foram utilizados por 3,0\% dos universitários, outros 3,0\% alguma vez já mascaram fumo ou aspiraram rapé e $8,0 \%$ consumiram charutos, cigarrilhas ou cachimbo, sendo que $6,9 \%$ desses com uma frequência mínima de 20 vezes.

Pode-se constatar que a exposiçáo tabagística ambiental, ou seja, quem fica em contato com a fumaça do cigarro em casa, trabalho ou faculdade representou 46,3\% dos universitários.

Quanto ao número de tabagistas que residem com os acadêmicos, $66,1 \%$ não convive com fumante em casa. Entretanto, o convívio com a fumaça do cigarro dentro de casa está presente na vida cotidiana de 67 universitários, dos quais 75,8\% convivem com uma pessoa que fuma dentro de casa, 20,9\% com duas e ainda $3,3 \%$ com três pessoas. 
A Tabela I apresenta a distribuição dos universitários, segundo os fumantes com quem convivem. Alguns alunos descreveram uma ou mais pessoas tabagistas no seu convívio social, sendo $16,5 \%$ a maioria dos amigos, $15,4 \%$ o pai ou responsável paterno, $13,6 \%$ a mãe ou responsável materna, $12,7 \%$ melhor amigo(a), 7,6\% seu irmão, e ainda $5,7 \%$ o companheiro(a).

Tabela I - Distribuição dos universitários, segundo os fumantes com quem convivem. São José do Rio Preto, 2014.

\begin{tabular}{lcc}
\hline \multicolumn{1}{c}{ Variáveis } & $\mathrm{N}$ & $\%$ \\
\hline Maioria dos amigos & 61 & 16,5 \\
Pai/responsável paterno & 57 & 15,4 \\
Mãe/responsável materna & 50 & 13,6 \\
Melhor amigo & 47 & 12,7 \\
Irmão & 28 & 7,6 \\
Companheiro(a) & 21 & 5,7 \\
\hline
\end{tabular}

Quando questionados sobre a afirmativa que relaciona os fumantes passivos de tempo prolongado ao desenvolvimento futuro de doenças tabaco-relacionadas e o possível óbito, $92,1 \%$ declararam a afirmativa verdadeira contra $2,7 \%$ que a consideraram falsa.

As opinióes dos universitários quanto à permissão do fumo em ambientes públicos ou de uso coletivo está retratado na Tabela II, que aponta que 91,3\% dos universitários acreditam que deve ser proibido fumar em ambientes de trabalho fechados, $90,2 \%$ proibido em instituição de saúde, $84,3 \%$ nas escolas, 78,6\% em restaurantes e, independente dos locais, $0,5 \%$ acha que o consumo de cigarro deve ser permitido em todas as áreas.

\section{Cessação do hábito de fumar}

Uma estratégia adotada pelo Ministério da Saúde, contra o tabagismo no Brasil, é a de estampar no maço de cigarros fotografias de pessoas com doenças relacionadas ao consumo do tabaco. Entretanto, quando questionados sobre o efeito dessa estratégia, 57,5\% dos universitários não acreditam que essas imagens estimulem a parar de fumar.

A iniciativa de cessar o tabagismo foi negada por $22,5 \%$ dos fumantes e $77,5 \%$ tiveram essa atitude uma vez. Destes, 48,0\% fizeram até três tentativas para cessar o hábito de fumar, $32,0 \%$, de quatro a seis tentativas e ainda 20,0\% abnegaram mais de 10 vezes. Para $25 \%$ dos universitários tabagistas, as tentativas para deixar de fumar aconteceram no último mês, enquanto que $61,0 \%$ experenciaram a abstinência há mais de cinco meses.

\section{Ex-fumantes}

Entre os 18 universitários ex-fumantes, 77,8\% cessaram o hábito de fumar há 5 anos, $11,1 \%$ entre $6 \mathrm{e}$ 7 anos, 5,5\% há mais de 10 anos e 22,1\% entre três e sete meses. Nessa população, 11,0\% fumaram durante um a cinco meses, $33,3 \%$ no período de um ano, $44,5 \%$ de dois a três anos, $11,0 \%$ de quatro a cinco anos.

Dentre os ex-fumantes, $50 \%$ afirmaram que fumavam cigarros diariamente, destes $83,3 \%$ consumiam de 1 a 6 cigarros/dia, 8,3\% 10 cigarros/dia e $8,3 \%$ fumavam 15 cigarros/dia.

Os 5,5\% dos universitários ex-fumantes pararam o consumo por algum problema de saúde causado pelo tabagismo ou porque piorou devido ao consumo, e estes receberam algum tipo de tratamento/acompanhamento de profissionais de saúde ou usaram algum medicamento para deixar de fumar; já os demais ex-fumantes $(94,5 \%)$ pararam por conta própria.

\section{Discussão}

Apesar de, no Brasil, o Ministério da Saúde promover campanhas antitabagismo, o consumo do tabaco é uma experiência que acontece geralmente na adolescência. Um estudo entre adolescentes e adultos da cidade de Belo Horizonte encontrou que um percentual de $51,0 \%$ dos sujeitos eram fumantes [18]. Na Colômbia, entre estudantes da enfermagem, a proporção de fumantes chega a $80 \%$ [19], valores

Tabela II - Distribuição das opiniões dos universitários da saúde quanto à permissão do fumo em ambientes públicos ou de uso coletivo. São José do Rio Preto, 2014.

\begin{tabular}{lcccccccc} 
& \multicolumn{2}{c}{ Restaurantes } & Instituições de saúde & & Escolas & \multicolumn{2}{c}{$\begin{array}{c}\text { Ambientes fechados } \\
\text { trabalho }\end{array}$} \\
\hline \multicolumn{1}{c}{ Áreas } & $\mathrm{N}$ & $\%$ & $\mathrm{~N}$ & $\%$ & $\mathrm{~N}$ & $\%$ & $\mathrm{~N}$ & $\%$ \\
\hline Todas & 2 & 0,5 & 2 & 0,5 & 2 & 0,5 & 2 & 0,5 \\
Algumas & 57 & 15,4 & 15 & 4,1 & 37 & 10,0 & 11 & 0,3 \\
Não permitido & 290 & 78,6 & 333 & 90,2 & 311 & 84,3 & 337 & 91,3 \\
\hline
\end{tabular}


superiores aos 43,6\% encontrados nesse estudo. Essa diferença, talvez, possa ser explicada pelas características culturais e sociais da regiáo do estudo, ou seja, uma cidade do interior do Estado de São Paulo.

Estudos apontam que os porcentuais de fumantes universitários da área da saúde, no Brasil, variam de $8,1 \%$ a $17,8 \%$, com destaque para a região sul, maior produtora de tabaco no Brasil [22-29]. No sudeste, o índice de tabagistas na população urbana é de 16,5\%, segundo o INCA [20].

Estudo entre acadêmicos de medicina aponta tempo médio de consumo de tabaco de 5,9 anos, corroborando os resultados deste estudo [29].

O maior consumo de cigarros industrializados, assim como o consumo diário de até 10 cigarros evidenciados neste estudo, é confirmado por outros estudos [25,26]. Embora alguns autores apontem que 70 a $80 \%$ dos estudantes consomem uma media de 10 cigarros/dia, em Lages/SC, o consumo médio chegou a 18 cigarros/dia para 58,6\% dos estudantes e, no curso de medicina de Santo Amaro/SP, a media diária de consumo diário foi de 20 cigarros para $91 \%$ dos estudantes [22,25,27-29].

Considerando-se que quanto maior o consumo de tabaco, maior é a nicotina-dependência que causa a compulsão de fumar, a dependência tabágica é um exemplo de doença crônica com remissóes e recaídas periódicas [1].

Para a OMS, o tabagismo cria uma "desordem mental e de comportamento em razão da síndrome da dependência à nicotina” [3]. Essa dependência pode ser verificada pelo tempo, após acordar, que o fumante consome o primeiro cigarro. Neste estudo foi de $68,0 \%$ na primeira hora, superando as taxas de $41,1 \%$ e ainda $67,0 \%$ com mais de 4 horas, apresentada em outros estudos [22,25].

A prevalência do tabagismo no sexo masculino é constatada pela maioria dos estudos sobre a temática, porém existe um aumento do consumo entre as mulheres [20,24,25,29]. Destacam-se, em escolas particulares, na regiáo do Distrito Federal, que as meninas de 9 a 19 anos se revelaram como maiores consumidoras de tabaco em relaçáo ao sexo masculino da mesma faixa etária [30].

Entre o consumo de produtos derivados do tabaco, Andrade et al. [26] apontam um índice de consumo de charuto e cachimbo por $8,0 \%$ dos universitários fumantes da Universidade de Brasília, índice superior ao encontrado neste estudo.

Alguns autores destacam que o convívio com fumantes é um fator de risco para o tabagismo, a presença de ter amigos, irmáo, namorado ou companheiro fumante aumenta em mais de seis vezes a chance de fumar, com destaque para irmão mais velho tabagista e a presença de mãe e/ou pai fumantes [23,25-27,31,32]. Estudando a percepção de estudantes universitários sobre o consumo de tabaco e a saúde, Silva et al. [33] mostram que os universitários associaram a palavra fumo à figura paterna em dois aspectos: um como o tabagista a ser copiado e outro de repressor do tabagismo, mostrando mais uma vez que o convívio com fumantes pode ser um importante fator de risco.

É consenso entre a maioria da população que o consumo de tabaco pode causar o desenvolvimento de doenças graves, sendo também uma verdade para 93,0\% dos fumantes descritos na pesquisa do INCA [20]. Essa conscientização faz com que a população acate as medidas antifumo em ambientes públicos [25].

A cessação do tabagismo é estimulada pelo Ministério da Saúde, com rótulos de advertências nos maços de cigarros, o que entre os universitários não surte muito efeito. Entretanto, segundo o INCA [20], pelo menos $65,0 \%$ dos fumantes já pensaram em parar de fumar, por sentirem-se sensibilizados pelas imagens.

Este estudo apontou a taxa de $1,1 \%$ de universitários ex-fumantes. Estudo do INCA [31] descreve que $50 \%$ dos fumantes gostariam de parar de fumar imediatamente e, embora tentem, não obtêm resultados.

\section{Conclusão}

A prevalência do tabagismo encontrada entre os universitários é semelhante a outros estudos e o convívio com fumantes intensificou esse vício. Porém, destaca-se que uma parcela dos alunos iniciou o consumo do tabaco na universidade. $\mathrm{O}$ tempo de consumo superior a 4 anos demonstra a necessidade de intensificação das açóes antifumo nas escolas, ou seja, antes do ingresso na universidade. Além disso, a intensificação dos programas antitabagismo entre universitários da área da saúde poderá contribuir para melhorar sua qualidade de vida e conscientizá-los de sua missáo futura que é promover a saúde da população.

\section{Referências}

1. World Health Organization (WHO). Report on the global tobacco epidemic, implementing smoke-free environments, 2009. WHO: Geneva; 2009.

2. Diário Oficial do Estado de São Paulo. Diário da Assembléia Legislativa No 71 - DOE de 16/04/08 - p.47. 
Projeto de Lei No 253, 2009. São Paulo: Diário Oficial do Estado de São Paulo; 2009.

3. Ministério da Saúde. Instituto Nacional de Câncer. Programa tabaco ou saúde. Rio de Janeiro: Ministério da Saúde; 2002.

4. World Health Organization (WHO). Brazil - Health Warnings on Tobacco Products Coordination for Cancer Prevention and Surveillance - Conprev Tobacco Control Division, 2009. Rio de Janeiro: Inca: 2009.

5. Oliveira AF, Valente JG, Leite IC. Aspectos da mortalidade atribuível ao tabaco: revisão sistemática. Rev Saúde Pública 2008;42(2):335-45.

6. Malbergier A, Oliveira Junior HP. Dependência de tabaco e comorbidade psiquiátrica. Rev Psiq Clín 2005;32(5)276-82.

7. Peuker AC, Fogaça J, Bizarro L. Expectativas e beber problemático entre universitários. Psicologia: Teoria e Pesquisa 2006;22(2):193-200.

8. Cunningham EC, Chaname E, Cunningham J, Brands B, Strike C, Wright MGM. Normas percibidas por los estudiantes universitarios de enfermería acerca de sus pares y el uso de drogas en Lima, Peru. Rev Latinoam Enferm 2009;17(Esp.):865-70.

9. Spiandorello WP, Filippini LZ, Dal Pizzol A, Kreische F, Soligo DS, Spiandorello T, et al. Avaliação da participaçáo de pequeno número de estudantes universitários em um programa de tratamento do tabagismo. Jornal Brasileiro Pneumologia 2007;33(1):69-75.

10. Oliveira EB, Furegato ARF. O trabalho do acadêmico de enfermagem como fator de risco para o consumo de álcool e outras drogas. Rev Latinoam Enferm 2008;16( n.spe):565-71.

11. Iglesias V, Cavada G, Silva C, Cáceres D. Consumo precoz de tabaco y alcohol como factores modificadores del riesgo de uso de marihuana. Rev Saúde Pública 2007;41(4):517-22.

12. Da Franca C, Colares V. Estudo comparativo de condutas de saúde entre universitários no início e no final do curso. Rev Saúde Pública 2008;42(3):420-7.

13. Ministério da Saúde. Inquérito domiciliar sobre comportamentos de risco e morbidade referida de doenças e agravos não transmissíveis: Brasil, 15 capitais e Distrito Federal, 2002-2003. Rio de Janeiro: Inca; 2004.

14. World Health Organization [homepage na Internet]. [acesso em 2014 Mar 3]. Who steps instrument (core and expanded). The WHO STEPwise approach to chronic disease risk factor surveillance. [citado 2014 Mar 3]. Disponível em URL: http://www.who.int/ chp/steps/STEPS_Instrument_v2.1.pdf

15. Centers for Disease Control and Prevention. Behavioral risk factor surveillance system survey questionnaire. Atlanta: Department of Health and Human Services; 2011.

16. Centers for Disease Control and Prevention. National Center for Health Statistics. The Third National Health and Nutrition Examination Survey (NHANES III, 1988-1994): reference manuals and reports. Maryland: Department of Health and Human Services; 1996.

17. International Physical Activity Questionnaire. Short last 7 days self-administered format. For use with young and middle-aged adults (15-69 years). [citado 2014 Mar 3]. Disponível em URL: http://www.sdprc. net/lhn-tools/IPAQ_SHORT_SELF_08_2002.pdf13.
18. Abreu MNS, Souza CF, Caiaffa WT. Tabagismo entre adolescentes e adultos jovens de Belo Horizonte, Minas Gerais, Brasil: influência do entorno familiar e grupo social. Cad Saúde Pública 2011;27(5):935-43.

19. SA, Vélez LF. Características del consumo de tabaco en estudiantes de enfermería de la Universidad de Antioquia (Colombia). Invest Educ Enferm 2010;28(3):370-83.

20. Ministério da Saúde. Inquérito domiciliar sobre comportamentos de risco e morbidade referida de doenças e agravos não transmissíveis: Brasil, 15 capitais e Distrito Federal, 2002-2003. Rio de Janeiro: Inca; 2004.

21. Centers for Disease Control and Prevention (CDC). Behavioral Risk Factor Surveillance System Survey Questionnaire. Atlanta: U.S. Department of Health and Human Services, Centers for Disease Control and Prevention; 2010.

22. Rodrigues Júnior JC, Ferraz SMR, Bruno RX. Prevalência e perfil de tabagistas universitários ingressantes de uma instituição de ensino superior. Pulmão RJ 2009;18(1):14-18.

23. Cid HP, Carvalho AMP. Percepción de los beneficios del consumo de drogas y barreras para su abandono entre estudiantes del área de salud. Rev Latinoam Enferm 2008;16(especial):621-6.

24. Azevedo e Silva G, Valente JG, Almeida LM, Moura EC, Malta DC. Tabagismo e escolaridade no Brasil, 2006. Rev Saúde Pública 2009;43(Supl 2):48-56.

25. Stramari LM, Kurtz M, Corrêa da Silva LC. Prevalência e fatores associados ao tabagismo em estudantes de medicina de uma universidade em Passo Fundo (RS). J Bras Pneumol 2009;35(5):442-8.

26. Andrade APA, Bernardo ACC, Viegas CAA, Ferreira DBL, Gomes TC, Sales MR. Prevalência e características do tabagismo em jovens da Universidade de Brasília. J Bras Pneumol 2006;32(1):23-8.

27. Menezes ANB, Hallal PC, Silva F, Souza M, Paiva L, D'Ávila A, et al. Tabagismo em estudantes de Medicina: tendências temporais e fatores associados. J Bras Pneumol 2004;30(3):223-8.

28. Kuhnen M, Kuhnen M, Boing AF, Oliveira MC, Longo GZ, Njaine K. Tabagismo e fatores associados em adultos: um estudo de base populacional. Rev Bras Epidemiol 2009;12(4):615-26.

29. Magliari RT, Pagliusi AL, Previero MB, Menezes FR, Feldman A, Novo NF. Prevalência de tabagismo em estudantes de faculdade de medicina. Rev Med (Sáo Paulo) 2008;87(4):264-71.

30. Rodrigues MC, Viegas CAA, Gomes EL, Morais JPMG, Zakir JCO. Prevalência do tabagismo e associação com o uso de outras drogas entre escolares do Distrito Federal. J Bras Pneumol 2009;35(10):986-91.

31. Instituto Nacional de Câncer (INCA). Inquérito "Vigilância de Tabagismo em Universitários da Área da Saúde". Divisão de Epidemiologia, Coordenação de Prevençáo e Vigilância. Rio de Janeiro: Inca; 2007.

32. Malcon MC, Menezes AMB, Chatkin M. Prevalência e fatores de risco para tabagismo em adolescentes. Rev Saúde Pública 2003;37:1-7.

33. Silva AO, Sousa CMM, Gaspar MFM, Paredes MAS, Tura LFR, Jesuino JC. Tabaco e saúde no olhar de estudantes universitários. Rev Bras Enferm 2008;61(4):423-7. 